\title{
Embedment of Nano-Graphene in Metal Deposits via Cold Spraying
}

\author{
Wen Sun ${ }^{1,2}$, Adrian Wei-Yee Tan ${ }^{1,2}$, Kaiqiang Wu ${ }^{1,2}$, Ayan Bhowmik ${ }^{1,2}$, Iulian Marinescu ${ }^{3}$, Erjia Liu ${ }^{2 *}$ \\ ${ }^{1}$ Rolls-Royce@NTU Corporate Lab, Nanyang Technological University, 50 Nanyang Avenue, Singapore \\ 639798, Singapore \\ ${ }^{2}$ School of Mechanical and Aerospace Engineering, Nanyang Technological University, 50 Nanyang \\ Avenue, Singapore 639798, Singapore \\ sunwen@ntu.edu.sg; adriantan@ntu.edu.sg; kaiqiang001@e.ntu.edu.sg; abhowmik@ntu.edu.sg \\ ${ }^{3}$ Civil Aerospace - Materials, Rolls-Royce Singapore Pte Ltd, 1 Seletar Aerospace Crescent, Singapore \\ 797565, Singapore \\ Iulian.Marinescu2@Rolls-Royce.com \\ * Corresponding author. Email: mejliu@ntu.edu.sg (E. Liu)
}

\section{Extended Abstract}

Nano-graphene (or "Graphene nanoplates (GNPs)") has been considered as an ideal reinforcement in metal matrix composites (MMCs) due to its unique two-dimensional characteristics. In recent years, many processes have been developed for fabrication of GNPs-metal matrix composites including spark plasma sintering [1], selective laser melting $[2,3]$, hot pressing [4], etc. However, these fabrication methods, for fabricating GNPs reinforced MMCs, mostly require extremely high processing temperature to melt the metal binders. This in turn significantly increases the risk of phase transformation, oxidation and thermal stress, thereby degrading the material performances. Cold spray is an emerging deposition technique which allows for the deposition of metal alloy materials and MMCs onto substrates at a very low temperature (well below the metal melting temperature) [5-6]. Contrary to the conventional thermal based methods, the feedstock powders remain solid-state during the cold spray deposition process without melting both the substrate and powder materials. Thus, usual problems like oxidation, phase transformation and thermal distortion can be avoided. In this study, the GNPs were embedded into Inconel 718 metal deposits via high pressure cold spray technique. The microstructure, mechanical property and tribological behaviour of the Inconel 718-GNPs composites were systemically investigated. Through process optimization, the Inconel 718-GNPs composites could be effectively synthesized with the incorporation of $0.15 \mathrm{wt} \%$ GNPs. The GNPs were uniformly distributed within the cold sprayed composite deposits, which were proved from microstructural observation and EDS analysis. The deposition efficiency gradually increased with decreasing GNPs content. Following this lead, the surface roughness of the as-sprayed composite deposits was found to be related to the addition of GNPs. The surface roughness gradually increased with increasing GNPs content and the composite deposit surfaces had plateau-like and crater-like features due to the dislodging of the weakly bonded particles. TEM lamellae were extracted from the composite deposits by focused ion beam milling. Through careful observation, it was found that the interface between the GNPs and the matrix was clean and well bonded, illustrating a mechanical bonding rather than the so-called chemical/metallurgical bonding. Besides, the TEM observations also indicated that the GNPs acted as a buffer layer preventing extreme deformation of the Inconel 718 splats with the GNPs themselves accommodating a significant portion of the impact energy during the cold spray deposition. Moreover, the Inconel 718GNPs composites provided appreciable reductions in coefficient of friction and wear rate due to several reasons. Firstly, the GNPs worked as a solid lubricant during the wear test process, which greatly decreased abrasion of the Inconel 718 matrix. Secondly, following the initial dislodging, the GNPs distributed along the sliding direction and stacked on the wear track surface thus forming a protective layer between the abrasive tip and the sample to prevent further wear. Thirdly, the GNPs had an exfoliated structure, and the GNP fragments adjacent to the Inconel 718 matrix surface provided an additional sliding effect due to separation of the GNP layers from one another. Hence, the GNPs-reinforced metal composites could be an excellent candidate for low friction and high wear-resistance applications. 


\section{References}

[1] Y. Song, Y. Chen, W. W. Liu, W. L. Li, Y. G. Wang, D. Zhao, X.B. Liu "Microscopic mechanical properties of titanium composites containing multi-layer graphene nanofillers," Mater. Des., vol. 109, pp. 256-263, 2016.

[2] Y. Wang, J. Shi, S. Lu, Y. Wang, "Selective laser melting of graphene-reinforced Inconel 718 superalloy: evaluation of microstructure and tensile performance," J. Manuf. Sci. Eng., vol. 139, pp. 1-6, 2017.

[3] W.H. Xiao, S.Q. Lu, Y.C. Wang, J. Shi, "Mechanical and tribological behaviors of graphene/Inconel 718 composites," Trans. Nonferrous Met. Soc. China, vol. 28, pp. 1958-1969, 2018.

[4] L. Wu, R. Wu, J. Zhang, L. Hou, "Synergistic effect of carbon nanotube and graphene nanoplatelet addition on microstructure and mechanical properties of AZ31 prepared using hot-pressing sintering," J. Mater. Res., vol. 33, pp. 4261-4269, 2018.

[5] W. Sun, A. Bhowmik, A.W.Y. Tan, R. Li, F. Xue, I. Marinescu, F. Li, E. Liu, "Improving microstructural and mechanical characteristics of cold-sprayed Inconel 718 deposits via local induction heat treatment," J. Alloys Compd., vol. 797, pp. 1268-1279, 2019.

[6] A.W.Y. Tan, W. Sun, A. Bhowmik, J.Y. Lek, I. Marinescu, F. Li, N.W. Khun, Z. Dong, E. Liu, "Effect of coating thickness on microstructure, mechanical properties and fracture behaviour of cold sprayed Ti6Al4V coatings on Ti6Al4V substrates," Surf. Coat. Technol., vol. 349, pp. 303-317, 2018. 